1/nstituto de Nutrición y Tecnología de los Alimentos (INTA), Universidad de Chile, Santiago, Chile.

${ }^{2}$ Instituto Clínico Oncológico Fundación Arturo López Pérez, Santiago, Chile. ${ }^{a}$ Nutricionista.

Recibido el 25 de octubre de 2013, aceptado el 15 de octubre de 2014

Correspondencia a: Dra. Loreto Plañella Instituto de Nutrición y Tecnología de los Alimentos (INTA), Universidad de Chile, Santiago, Chile e Instituto Clínico Oncológico Fundación Arturo López Pérez, Santiago, Chile. loreto.panella@falp.org

\section{Relación entre estado nutricional y evolución postoperatoria, en cirugía oncológica digestiva}

\author{
LORETO PAÑELLA $^{1,2}$, MARLENE JARA $^{1}$, MORELIA CORNEJO ${ }^{2, a}$, \\ XIMENA LASTRA ${ }^{2, a}$, MARÍA GLADYS CONTRERAS ${ }^{2, a}$, \\ KATTIA ALFARO ${ }^{2, a}$, MARÍA PÍA DE LA MAZA ${ }^{1,2}$
}

\section{Nutritional status and postoperative complications in patients with digestive cancer}

Background: Risk of malnutrition is elevated among oncologic patients, and this increases postoperative morbidity and mortality. Aim: To study the association between nutritional status and postoperative outcomes in a group of patients with gastrointestinal cancers. Patients and Methods: We studied 129 patients with diagnosis of digestive cancer, previous to potentially curative surgery. Nutritional status was evaluated through anthropometric measures, Subjective Global Assessment (SGA), dietary intake recalls and routine biochemical parameters. Functional performance was assessed by the Karnofsky index (KI). Cancer stage was classified according to TNM4. During the postoperative period, complications, length of stay at the critical care ward and duration of hospitalization were registered. Thirty days after discharge, patients were contacted, and the appearance of new complications was listed. Results: According to SGA $14.7 \%$ of patients were classified as well nourished (A), $57.3 \%$ as moderately undernourished or at risk of malnutrition (B) and $27.9 \%$ as severely malnourished (C). The incidence of total complications was $25.5 \%$. Nutritional status was not associated with cancer stage. The frequency of complications among patients classified as $A, B$ and $C$ were $5.5,25.3$ and $37.1 \%$ respectively ( $p=0.03)$. Conclusions: We detected a high frequency of malnutrition in this group of patients. Overall the frequency of postoperative complications was low, however malnourished patients exhibited a higher rate of surgical complications.

(Rev Med Chile 2014; 142: 1398-1406)

Key words: Medical Oncology; Nutrition assessment; Surgery.
L a desnutrición y pérdida de peso es un problema frecuente en los pacientes con cáncer, afectando el pronóstico, prolongando estadías hospitalarias, encareciendo los costos y afectando la calidad de vida y la sobrevida ${ }^{1}$. Esto es consecuencia del "síndrome caquexia anorexia", cuyos criterios diagnósticos fueron establecidos recientemente ${ }^{2}$. La prevalencia de este síndrome alcanza a 40 a $80 \%$ de los pacientes durante el curso de su enfermedad y varía según el tipo de tumor, localización y estadío de la enfermedad ${ }^{3,4}$.
Las causas son múltiples y van desde los efectos directos del tumor en el tubo digestivo, hasta aquellos efectos indirectos secundarios al tratamiento oncológico ${ }^{5}$. Las alteraciones metabólicas inducidas por el tumor generan una respuesta inflamatoria sistémica con liberación de citoquinas (factor de necrosis tumoral, interleuquinas 1 y 6, interferón gamma), las que están implicadas en el catabolismo proteico, depleción muscular y consecuente pérdida de funcionalidad ${ }^{6}$.

Por otro lado, aunque el estadío del tumor, ha 
sido considerado como un factor principal para predecir la sobrevida en pacientes que se someten a cirugía por cáncer, es evidente que las complicaciones postoperatorias podrían contribuir a disminuir la sobrevida de estos pacientes ${ }^{7-9}$. En este sentido, numerosos estudios confirman el impacto negativo de la desnutrición, o la falta de soporte nutricional, en las complicaciones quirúrgicas $^{10-15}$, potencialmente prevenibles a través de intervenciones específicas ${ }^{16-20}$.

La evaluación nutricional de los pacientes hospitalizados, y específicamente los pacientes oncológicos, es compleja, debido a múltiples factores que interfieren en las mediciones antropométricas y bioquímicas. Existe baja concordancia entre los distintos criterios diagnósticos usados para evaluar desnutrición ${ }^{21}$ y no existe consenso en cuál método usar para evaluar el estado nutricional en pacientes con cáncer ${ }^{21}$. Las Guías Clínicas de la Sociedad Europea de Nutrición Clínica y Metabolismo (ESPEN) recomiendan el Nutritional Risk Sreening (NRS 2002), para evaluación nutricional de pacientes hospitalizados, sin embargo, éste aún no ha sido validado en cohortes de pacientes con enfermedad neoplásica avanzada. Tanto la evaluación global subjetiva (EGS) como la EGS generada por el paciente (EGS GP) $)^{22}$ no incluyen datos objetivos y cuantificables, pero son herramientas válidas en la evaluación de los pacientes con cáncer $^{23-28}$ y se correlacionan bien con los métodos clásicos, constituyendo excelentes indicadores de pronóstico ${ }^{29}$.

El objetivo principal del presente estudio fue evaluar morbimortalidad postoperatoria en un grupo de pacientes oncológicos digestivos, y su relación con estado nutricional preoperatorio.

\section{Pacientes y Métodos}

\section{Pacientes}

Se incluyeron pacientes de la Fundación Arturo López Pérez (FALP), mayores de 18 años, portadores de cáncer digestivo, con indicación quirúrgica. Se etapificó los tumores según la clasificación $\mathrm{TNM}^{30}$. Se excluyeron aquellos pacientes bajo tratamiento con quimioterapia $(\mathrm{QT})$, radioterapia (RT) o corticoides al momento de la evaluación y aquellos con enfermedad fuera de alcance terapéutico curativo. Este estudio contó con la aprobación de los comités de ética de la FALP y del Instituto de Nutrición y Tecnología de los Alimentos (INTA).
Luego de firmar un consentimiento informado, en cada paciente se efectuó una evaluación del estado nutricional a través de EGS, cálculo del porcentaje de pérdida de peso con respecto al peso habitual en los últimos 6 meses, parámetros antropométricos (peso, talla, perímetro braquial $[\mathrm{PB}]$ y pliegue cutáneo tricipital $[\mathrm{PCT}]$ ), calculando área muscular braquial a través de la fórmula $[\mathrm{AMB}$ $\left.\left.=\mathrm{PMB}^{\star} 2 / 4^{\star} 3,14\right]\right)^{31}$, y parámetros bioquímicos (proteínas totales, albúmina, recuento de linfocitos y colesterol total). Además se cuantificó la ingesta dietaria a través de encuestas (recordatorio de 24 h y tendencia de consumo), especificando si el paciente había recibido alguna suplementación nutricional previa al ingreso.

El nivel de funcionalidad se evaluó a través del índice de Karnofsky (IK) y como indicador de inflamación asociada a la neoplasia se midió proteína $\mathrm{C}$ reactiva (PCR). Las determinaciones de laboratorio fueron efectuadas con técnicas automatizadas en el laboratorio clínico de la FALP.

Posteriormente, en cada caso se verificó el tipo de cirugía efectuada, tiempo operatorio, sangrado y transfusiones intraoperatorias. Luego se efectuó un seguimiento de la evolución postoperatoria, registrando la aparición de complicaciones en base a las definiciones establecidas ${ }^{15}$, dividiéndolas entre mayores y menores, infecciosas y no infecciosas (Tabla 1). Se registró tiempo de permanencia en UCI y tiempo de hospitalización total. Treinta días después del alta, los pacientes fueron controlados ambulatoriamente o llamados telefónicamente, para documentar la aparición de nuevas complicaciones o reingresos y la etapificación del tumor resecado.

Los datos se expresaron como promedio \pm desviación estándar o mediana (rango) según la distribución de las variables. Para efectuar comparaciones de las variables continuas entre los grupos se utilizó análisis de varianza o Kruskall Wallis respectivamente. Para analizar las diferencias en frecuencia de las variables categóricas se utilizó el test de probabilidad exacta de Fisher. El nivel de significación se fijó en $\mathrm{p}<0,05$. Todos los análisis estadísticos se efectuaron en el programa Stata 10.1.

\section{Resultados}

Se preseleccionaron 147 pacientes, excluyendo 19 (18 casos por detectarse tumor no resecable 


\section{Tabla 1. Definición de complicaciones}

\begin{tabular}{|c|c|}
\hline & Definición \\
\hline \multicolumn{2}{|c|}{ Complicaciones infecciosas mayores } \\
\hline Neumonía & Fiebre $>30^{\circ} \mathrm{C}$, radiografía de tórax positiva, que requiera tratamiento antibiótico \\
\hline Absceso abdominal & $\begin{array}{l}\text { Gran colección purulenta intraabdominal, confirmada imagenológicamente que requiera } \\
\text { antibiótico y drenaje }\end{array}$ \\
\hline Sepsis & $\begin{array}{l}\text { Síndrome de respuesta inflamatoria sistémica que es la presencia de } 2 \text { o más de: Recuento } \\
\text { de blancos }>12.000 \text { (o menos de } 4.000 \text { ), temperatura superior a } 38^{\circ} \mathrm{C} \text { (o inferior a } 36^{\circ} \text { ), FC } \\
>90 / \mathrm{min}, \mathrm{FR}>20 / \mathrm{min} \text {, foco infeccioso, bacterias aisladas de cualquier sitio }\end{array}$ \\
\hline Bacteriemia & Bacteriemia: dos hemocultivo positivos consecutivos sin compromiso hemodinámico \\
\hline Fasceitis necrotizante & $\begin{array}{l}\text { Infección que afecta la piel, tejido celular subcutáneo, y sistema músculo aponeurótico, acom- } \\
\text { pañado de compromiso séptico sistémico. Requiere de tratamiento antibiótico y quirúrgico }\end{array}$ \\
\hline Cuagulopatia séptica & Tiempo de protrombina prolongada o plaquetopenia inferior a 100.000/ml \\
\hline \multicolumn{2}{|c|}{ Complicaciones Infecciones menores } \\
\hline Infección de la herida & Enrojecimiento o sensibilidad de la herida quirúrgica con la secreción purulenta \\
\hline Infección del tracto urinario & Urocultivo con $>100.000 \mathrm{Ufc} / \mathrm{ml}$ de orina \\
\hline \multicolumn{2}{|c|}{ Complicaciones no infecciosas mayores: } \\
\hline $\begin{array}{l}\text { Dehiscencia anastomótica } \\
\text { con o sin fístula }\end{array}$ & $\begin{array}{l}\text { Salida de contenido intestinal y/o gases a través de una sutura de la herida principal o la fistu- } \\
\text { lización a un órgano vecino; con hallazgos de una re intervención por peritonitis secundaria } \\
\text { a colección o una filtración; pudiendo ser evidenciada por TAC, laparotomía exploratoria, } \\
\text { ecografía }\end{array}$ \\
\hline Dehiscencia de herida & Rotura parcial o total de cualquiera de las capas de la herida quirúrgica \\
\hline Hemorragia & De cualquier origen que requiera transfusión de sangre \pm 2 unidades \\
\hline \multicolumn{2}{|c|}{$\begin{array}{l}\text { Perforación gastrointestinal, } \\
\text { obstrucción, e isquemia }\end{array}$} \\
\hline Pancreatitis & $\begin{array}{l}\text { Definida por clínica, niveles sérico elevados de amilasa y lipasa, además de imagenología } \\
\text { concordante }\end{array}$ \\
\hline Infarto al miocardio & Comprobado por ECG, ecocardiograma y CK-MB sérica elevada o troponina I \\
\hline Shock cardiogénico & $\begin{array}{l}\text { Diagnóstico }(\mathrm{Dg}) \text { clínico, Presión sistólica }<90 \mathrm{mmHg} \text { por más de } 30 \mathrm{~min} \text {, baja de TA en } 10 \\
\text { puntos del basal, Índice cardiaco }<2,2 \mathrm{~L} / \mathrm{min} / \mathrm{m} 2 \text {, presión en cuña mayor de } 18 \mathrm{mmHg} \text {, Dg } \\
\text { por ecocardiograma y electrocardiograma y radiografía de tórax }\end{array}$ \\
\hline Embolia pulmonar & $\begin{array}{l}\text { Según clínica, confirmación por imagenología ( } \mathrm{Rx} \text {, angiotac), requerimiento de tratamiento } \\
\text { (heparinización o fibrinolíticos) }\end{array}$ \\
\hline Hemoperitoneo & $\begin{array}{l}\text { Dg definido por la clínica (dolor, distensión abdominal con o sin compromiso hemodinámi- } \\
\text { co), descenso del hematocrito, diagnóstico por lavado peritoneal o punción, laparoscopia, } \\
\text { ecotomografía o TAC }\end{array}$ \\
\hline Insuficiencia respiratoria & Presencia de la disnea y la frecuencia respiratoria $>35 / \mathrm{min} \circ \mathrm{PaO}_{2}<70 \mathrm{mmHg}$ \\
\hline Insuficiencia circulatoria & Presión arterial inestable que requiera el uso extra de líquidos y / o estimulantes cardiacos \\
\hline Falla renal & Necesidad de hemodiálisis \\
\hline Insuficiencia hepática aguda & Aumento de la bilirrubina sérica o el nivel de enzimas hepáticas ( $50 \%$ encima del valor inicial). \\
\hline Disfunción renal & Incremento del nivel de creatinina o urea sérica ( $50 \%$ por encima del nivel basal). \\
\hline Fístula pancreática & $\begin{array}{l}\text { Producción diaria de líquido }>10 \mathrm{ml} \text { a través de un drenaje quirúrgico, con amilasa sérica } 5 \\
\text { veces superior a la concentración normal. }\end{array}$ \\
\hline $\begin{array}{l}\text { Síndrome de Disfunción } \\
\text { Orgánica Múltiple (MODS) }\end{array}$ & $\begin{array}{l}\text { Desarrollo progresivo de una disfunción de dos o más sistemas orgánicos, incapaz de mantener } \\
\text { la homeostasis }\end{array}$ \\
\hline \multicolumn{2}{|c|}{ Complicaciones no infecciosas menores } \\
\hline Derrame pleural & $\begin{array}{l}\text { Acumulo anormal de líquido en la cavidad torácica, diagnostico clínico y radiológico, siendo } \\
\text { complicación mayor si genera insuficiencia respiratoria }\end{array}$ \\
\hline $\begin{array}{l}\text { Trombosis venosa } \\
\text { profunda }\end{array}$ & Cualquier trombo en el sistema venoso profundo probado por angiotac \\
\hline
\end{tabular}


intraoperatoriamente y 1 paciente que rechazó la cirugía). En uno de los casos incluidos se efectuó una pancreatectomía subtotal, pero la biopsia resultó negativas para cáncer. Así la muestra final quedó constituida por 129 pacientes con tumores del tubo digestivo o sistema hepático-biliar, con edad de 60,9 \pm 11 años, 59 hombres y 70 mujeres. Se clasificó como cirugías digestivas altas $(n=63)$ a las resecciones de esófago, estómago, intestino delgado, páncreas, hepático-biliar y como cirugías digestivas bajas $(n=66)$ a las resecciones de recto y colon (Tabla 2). Además, 32 pacientes fueron tratados con RT y/o QT neoadyuvante durante los meses previos, la mayor parte de éstos ${ }^{24}$ correspondían a cánceres de colon y recto. Todas las cirugías fueron efectuadas por el mismo equipo quirúrgico, dirigido por dos cirujanos.

En la Tabla 3 se muestran las variables nutricionales y exámenes de laboratorio de los pacientes, divididos según la EGS, que se efectuó

Tabla 2. Distribución de las cirugías efectuadas $(n=129)$

\begin{tabular}{|llr|}
\hline Grupo quirúrgico & Cirugía efectuada & $\mathbf{n}$ \\
Digestivo alto & Resección Esófago o esófago-gástrica & 6 \\
& Resección gástrica total o subtotal & 26 \\
& Resección páncreas o duodeno-páncreas & 16 \\
& Resección intestino delgado & 1 \\
Total & Resección de lóbulo o segmento hepático & 15 \\
Digestivo bajo & & 64 \\
& Resección total o parcial de colon & 39 \\
Total & Resección anterior baja de recto & 22 \\
& Operación de Miles o exanteración pelviana & 4 \\
\hline
\end{tabular}

Tabla 3. Variables según estado nutricional determinado por EGS

\begin{tabular}{|c|c|c|c|c|}
\hline \multirow[t]{2}{*}{ Variables } & \multicolumn{3}{|c|}{ EGS } & \multirow[t]{2}{*}{$\mathbf{p}$} \\
\hline & A $n=18$ & B $n=75$ & C $n=35$ & \\
\hline Edad (años) & $58 \pm 14$ & $62 \pm 10$ & $60 \pm 12$ & 0,309 \\
\hline Peso $(\mathrm{kg})$ & $72(55-96)$ & $68(40-123)$ & $63(40-91)$ & $\begin{array}{c}0,005 \\
A \neq C \text { y } B \neq C\end{array}$ \\
\hline $\mathrm{IMC}\left(\mathrm{k} / \mathrm{m}^{2}\right)$ & $28(23-33)$ & $27(17-44)$ & $24(16-34)$ & $\begin{array}{c}0,0001 \\
A \neq C \text { y } B \neq C\end{array}$ \\
\hline Pérdida de peso (\%) & $0(0-13,6)$ & $4,8(0-18,3)$ & $13,8(4,5-32)$ & $\begin{array}{l}0,0001 \\
A \neq B \neq C\end{array}$ \\
\hline Pliegue tricipital (mm ) & $25,9 \pm 7,4$ & $21,7 \pm 8,9$ & $16,7 \pm 8,0$ & $\begin{array}{c}0,0008 \\
A \neq C \text { y } B \neq C\end{array}$ \\
\hline $\mathrm{AMB}\left(\mathrm{mm}^{2}\right)$ & $4.890 \pm 1.293$ & $4.590 \pm 1.124$ & $4.157 \pm 1.223$ & 0,0788 \\
\hline Índice de Karnofsky & $100(60-100)$ & $90(70-100)$ & $80(50-100)$ & $\begin{array}{l}0,0001 \\
A \neq B \neq C\end{array}$ \\
\hline Hemoglobina (g/dl) & $14(6-15)$ & $13(8-16)$ & $12(5-16)$ & $\begin{array}{c}0,006 \\
A \neq C \text { y } B \neq C\end{array}$ \\
\hline Albúmina (g/dL) & $4,2(3,5-4,7)$ & $4,1(2,1-5)$ & $4,0(2,9-4,7)$ & 0,079 \\
\hline$P C R(g / d L)$ & $0,2(0,1-3,2)$ & $0,3(0-7,0)$ & $0,5(0,01-8,8)$ & 0,228 \\
\hline Recuento de Linfocitos $\left(\mathrm{n} / \mathrm{mm}^{3}\right)$ & $1703 \pm 1006$ & $1665 \pm 768$ & $1613 \pm 562$ & 0,914 \\
\hline
\end{tabular}

$\mathrm{EGS}=$ evaluación global subjetiva, $\mathrm{IMC}=$ índice de masa corporal, $\mathrm{AMB}=$ área muscular braquial, $\mathrm{PCR}=$ proteína $\mathrm{C}$ reactiva cuantitativa. 
en 128 pacientes. Según ésta, $14,7 \%$ se encontró bien nutrido (A), 57,3\% presentaba desnutrición moderada (B) y 27,9\% desnutrición severa (C). Los pacientes desnutridos habían perdido más peso y tenían significativamente menor IK y PCT pero la diferencia en $\mathrm{AMB}$ no alcanzó significación estadística. Entre las variables de laboratorio clínico, sólo la hemoglobina resultó significativamente menor en el grupo C. Los niveles de albúmina sérica estaban dentro del rango normal en la mayor parte de los pacientes $(4,1[2,1-5,0]$ $\mathrm{g} / \mathrm{dL})$, mientras que el recuento de linfocitos (1.618 [132-3.864] $\left./ \mathrm{mm}^{3}\right)$ y valores de PCR $(0,35$ $[0-8,8] \mathrm{g} / \mathrm{dL})$ mostraron rangos muy amplios, sin alcanzar diferencias estadísticas según EGS. Si se clasifica como desnutridos a aquellos pacientes que perdieron $5 \%$ o más de su peso habitual (dato obtenido en 120 pacientes), se observó que ellos presentaron valores de IMC, $\mathrm{PB}, \mathrm{AMB}$, hemoglobina y albúmina significativamente menores, destacando además que el IK en este grupo resultó también significativamente menor (100 [70-100] versus 90 [50-100], $\mathrm{p}=0,0001$ ).

No se observaron diferencias estadísticamente significativas en las variables nutricionales ni en la funcionalidad según el estadío tumoral. Los pacientes en etapas más avanzadas tenían PCR significativamente más altas, pero la mayor parte de los valores se encontraron dentro de rangos normales (Tabla 4).

La ingesta alimentaria preoperatoria fue muy variable; 1.505 (600-3.492) Kcal/día y 59,5 (20139) g prot/día, sin diferencias significativas entre los cánceres digestivos altos y bajos. La ingesta alimentaria no se asoció a la etapa tumoral ni a estado nutricional evaluado a través de EGS, antropometría, ni a variables de laboratorio. En cuanto a suplementación nutricional preoperatoria, sólo 26 pacientes recibieron un suplemento oral, 16 de éstos del grupo digestivo alto y 10 del grupo digestivo bajo; 17 pacientes ingirieron Supportan Drink ${ }^{\circledR}$ (Fresenius-Kabi) $600 \mathrm{ml} /$ día + Glutamine Plus ${ }^{\circledR}$ (Fresenius- Kabi) 30 g/día y el resto recibió diferentes volúmenes de otras fórmulas poliméricas o concentrados proteicos, como Ensure ${ }^{\circledR}$ (Abbott Nutrition) y/o Proteinex ${ }^{\circledR}$ (Victus). Los pacientes suplementados tenían menor IMC y niveles de albúmina, pero un porcentaje de pérdida de peso similar a los enfermos no suplementados $(5,5 \%$ [0-28\%] versus 6,3\% [0-32\%] $\mathrm{p}=\mathrm{NS})$. El IK y demás variables estudiadas resultaron similares al comparar ambos grupos (datos no mostrados).

El tiempo operatorio promedió 3,5 $\pm 1,6 \mathrm{~h}$, fue superior en las cirugías digestivas altas comparado con las cirugías del tubo digestivo bajo $(4 \mathrm{~h}[1-8,5]$ versus $2,5 \mathrm{~h}[1,1-6], \mathrm{p}=0,0001)$. De igual forma,

Tabla 4. Variables según etapa tumoral

\begin{tabular}{|lccccc|}
\hline Variables & $\begin{array}{c}\mathbf{I} \\
(\mathbf{n}=\mathbf{1 7})\end{array}$ & $\begin{array}{c}\text { II } \\
(\mathbf{n}=\mathbf{3 3})\end{array}$ & $\begin{array}{c}\text { III } \\
(\mathbf{n}=\mathbf{5 1})\end{array}$ & $\begin{array}{c}\text { IV } \\
(\mathbf{n}=\mathbf{2 7})\end{array}$ & p \\
EDAD & $60,2 \pm 7,5$ & $58,0 \pm 13,9$ & $63,5 \pm 10,7$ & $60,4 \pm 11,0$ & 0,192 \\
EGS (n pacientes A/B/C) & $4 / 12 / 1$ & $3 / 20 / 10$ & $7 / 31 / 12$ & $4 / 11 / 12$ & 0,115 \\
Peso (k) & $65,0(50-88)$ & $66,6(42-92)$ & $69,0(40-96)$ & $69,0(45-123)$ & 0,402 \\
IMC (k/m²) & $26,0(20,8-32,1)$ & $26,6(16,2-41,8)$ & $26,5(16,6-43,7)$ & $25,0(17,2-40,4)$ & 0,994 \\
Disminución de peso (\%) & $4,4(0-19,3)$ & $4,7(0-27,7)$ & $6,4(0-32)$ & $10,7(0-26,9)$ & 0,079 \\
Pliegue Tricipital (mm) & $22,5 \pm 8,1$ & $21,7 \pm 9,3$ & $19,4 \pm 8,5$ & $25,1 \pm 9,2$ & 0,453 \\
AMB & $4.464 \pm 1.081$ & $4.306 \pm 1.094$ & $4.709 \pm 1.222$ & $4.530 \pm 1.301$ & 0,513 \\
Indice de Karnofsky & $90(70-100)$ & $90(70-100)$ & $90(70-100)$ & $90(50-100)$ & 0,168 \\
Hemoglobina (g/dl) & $12,5 \pm 1,9$ & $12,7 \pm 1,4$ & $12,1 \pm 2,4$ & $11,9 \pm 2,8$ & 0,727 \\
Albúmina (g/dL) & $4,2(3,7-4,7)$ & $4,1(3,0-4,6)$ & $4,0(2,9-4,5)$ & $4,0(2,1-5,0)$ & 0,187 \\
PCR (g/dL) & $0,1(0-4,9)$ & $0,2(0-8,2)$ & $0,5(0,03-8,8)$ & $0,7(0,01-7,1)$ & 0,019 \\
Recuento de Linfocitos (N/mm²) & $1.690 \pm 838$ & $1.820 \pm 756$ & $1.617 \pm 743$ & $1.519 \pm 674$ & 0,587 \\
\hline
\end{tabular}

$\mathrm{EGS}=$ evaluación global subjetiva, $\mathrm{IMC}=$ índice de masa corporal, $\mathrm{AMB}=$ área muscular braquial, $\mathrm{PCR}=$ proteína $\mathrm{C}$ reactiva cuantitativa. 
los pacientes del grupo digestivo alto presentaron mayor sangrado intraoperatorio que las cirugías digestivas bajas $(200 \mathrm{cc}$ [0-4.000] versus $50 \mathrm{cc}$ [0$1.000], \mathrm{p}=0,003)$ y permanecieron más tiempo en la UCI (2 [0-10] versus 2 [0-32] días, $\mathrm{p}=0,0002)$.

La estadía hospitalaria fue de 8 días [4-89], significativamente más larga entre los pacientes sometidos a cirugía digestiva alta con respecto a la cirugía del tubo digestivo bajo ( 9 días [4-89] versus 8 días [4-37]) $(\mathrm{p}=0,005)$. El tiempo de hospitalización promedio entre los desnutridos (EGS B y C) fue levemente superior comparado con los bien nutridos (10,2 \pm 9 versus 7,9 \pm 2 días, $p=N S)$. Los pacientes con estadios tumorales más avanzados no permanecieron más tiempo hospitalizados que aquellos con tumores menos extendidos.

En lo referente a evolución postoperatoria, durante la hospitalización se registraron 18 complicaciones, 10 de estas mayores (hemoperitoneo, absceso intra-abdominal, peritonitis, sepsis, dehiscencia de sutura) falleciendo 1 enfermo producto de una peritonitis. Entre las 8 complicaciones intrahospitalarias menores se registraron derrames pleurales o hemotórax de baja cuantía e íleos paralíticos. Un mes después del alta se sumaron 15 complicaciones menores más (predominando infecciones urinarias, seromas, infecciones de herida operatoria). En suma, de las 33 complicaciones registradas (incidencia 25,6\%), 16 eventos fueron considerados como complicaciones mayores $(48,5 \%)$. La frecuencia de complicaciones totales fue significativamente superior entre los pacientes desnutridos $(\mathrm{p}=0,037)$ (Tabla 5$)$, sin diferencias según estadío tumoral ni localización de la cirugía. La frecuencia de complicaciones fue levemente inferior entre los pacientes suplementados preoperatoriamente $(15,8$ versus $28 \%)$, sin alcanzar significación estadística.

Tabla 5. Complicaciones totales según estado nutricional evaluado por EGS

\begin{tabular}{|ccc|}
\hline EGS A & $\begin{array}{c}\text { Pacientes con } \\
\text { complicaciones }\end{array}$ & $\begin{array}{c}\text { Pacientes sin } \\
\text { complicaciones }\end{array}$ \\
EGS B & 1 & 18 \\
EGS C & 13 & 55 \\
$\begin{array}{c}\text { P según test } \\
\text { de Fisher }\end{array}$ & $\mathbf{0 , 0 3 0}$ & 22 \\
\hline
\end{tabular}

El 52\% de las complicaciones totales eran de carácter infeccioso, las cuales fueron significativamente más frecuentes en las cirugías del tubo digestivo bajo $(p=0,036)$. Las infecciones no resultaron más elevadas entre los desnutridos o pacientes en etapas tumorales más avanzadas.

Como factores asociados a la presencia de complicaciones postoperatorias totales en este estudio, se detectó correlaciones significativas con la duración de la cirugía $(\mathrm{rho}=0,26 \mathrm{p}=0,003)$ y con los días de ayuno postoperatorio ( $\mathrm{rho}=0,27$ $\mathrm{p}=0,002)$. Los factores asociados a infecciones fueron edad (rho $=-0,18 \mathrm{p}=0,04)$ y cirugía del tubo digestivo bajo.

\section{Discusión}

En este estudio, efectuado en un centro oncológico privado, que opera un volumen de pacientes importante, con buenos resultados post quirúrgicos y baja morbimortalidad, los resultados más relevantes fueron, por una parte cuestionar el concepto de que mientras más avanzada la etapa tumoral se observa un creciente y evidente compromiso nutricional, y por otra parte, constatar nuevamente que el deterioro nutricional eleva el riesgo de complicaciones.

La falta de correlación entre estadío oncológico y estado nutricional puede atribuirse en parte a lo limitado de los métodos de evaluación nutricional que están disponibles para uso clínico, por lo cual seguimos dependiendo de un método subjetivo pero validado, la EGS. En los últimos años se ha enfatizado en el concepto del síndrome de caquexia-anorexia por cáncer, los comités de expertos han establecido los criterios diagnósticos y puntos de corte para su detección. Sin embargo, no hemos logrado sensibilizar a los equipos oncológicos acerca de la necesidad de cuantificar la masa y función muscular objetivamente, antes de iniciar el tratamiento oncológico, porque éste habitualmente agrava el deterioro nutricional, transitoria o permanentemente. Por otra parte, la EGS se establece tomando en cuenta datos de la historia clínica, especialmente baja de peso, que es una de las bases sobre las cuales se establece el diagnóstico de precaquexia (reducción $<5 \%$ del peso) o caquexia (reducción $>5 \%)^{2}$ por lo cual es esperable que el diagnóstico de caquexia coincida con una EGS B o C. Sin embargo, muchos pa- 
cientes pueden ser obesos y presentar sarcopenia asociada al cáncer, que es difícil de detectar si no se cuenta con el equipamiento necesario (bioimpedanciometría, densitometría corporal, tomografía computada, etc), conduciendo al equipo oncológico tratante a considerar estos pacientes como bien nutridos y no preocuparse del soporte nutricional en forma activa. En este sentido, varios estudios han demostrado la utilidad de la tomografía computada para la evaluación de la pérdida de músculo esquelético en los pacientes con cáncer y su asociación con menor sobrevida ${ }^{32-34}$. En una reciente revisión del European Palliative Care Research Collaborative (EPCRC), incorporan la sarcopenia como criterio diagnóstico de caquexia por cáncer, junto con la pérdida de peso y el índice de masa corporal (IMC) $)^{2}$. En nuestra muestra el $60,5 \%$ de los pacientes tenía IMC $>25 \mathrm{k} / \mathrm{m}^{2}$ y observamos una relación inversa entre IMC y \% de baja de peso, por lo cual incluso el equipo investigador podría haber subestimado el real compromiso muscular y funcional de estos pacientes. Al repetir los análisis en 120 pacientes que conocían su peso previo, clasificando con caquexia ( $\geq 5 \%$ pérdida de peso) o sin caquexia ( $<5 \%$ reducción de peso), tampoco detectamos asociación con estadío tumoral ni mayor frecuencia de complicaciones quirúrgicas entre los primeros.

Dicho esto, utilizando EGS obtuvimos las cifras esperables para un centro oncológico, es decir, una minoría de los pacientes se pueden clasificar como bien nutridos, predominando los B y C. Sin embargo, en nuestra muestra, a pesar de existir asociación entre EGS y albúmina, IK y PCR, los valores estaban muy cerca de la normalidad, sugiriendo que probablemente estos pacientes consultaron precozmente, antes de presentar síntomas y compromiso funcional más marcado. Otra posible explicación es que la EGS pueda sobreestimar la real prevalencia de desnutrición ${ }^{21}$. A pesar de ello, los pacientes clasificados como desnutridos según EGS presentaron una frecuencia más alta de complicaciones postoperatorias y una estadía hospitalaria levemente superior ( $\sin$ alcanzar significación estadística). Cabe destacar que la incidencia de complicaciones fue reducida, predominando aquellas de bajo riesgo, pero que se fueron sumando al prolongar el período de seguimiento hasta después del alta hospitalaria.

En los escasos pacientes en que se logró indicar un suplemento enteral por vía oral durante una semana antes de la cirugía, observamos una reducción en las complicaciones postoperatorias totales, a pesar de que estos enfermos presentaban mayor deterioro nutricional. Obviamente dado el reducido número de casos las diferencias no alcanzaron significación estadística. A pesar de las recomendaciones de la ASPEN y ESPEN ${ }^{35}$, esta práctica aún no se ha instituido de rutina, pero nuestros pocos casos sugieren que puede ser de utilidad, independiente del estado nutricional y estadío tumoral. Por lo tanto, queda como tarea a futuro verificar si el soporte preoperatorio puede determinar menores complicaciones postoperatorias.

En conclusión nuestros datos cuestionan la relación entre EGS y estadío tumoral, lo cual hace más patente la necesidad de contar con métodos más objetivos para diagnosticar caquexia por cáncer. Sin embargo, aún utilizando esta herramienta subjetiva pero ampliamente validada, encontramos que los pacientes con deterioro nutricional presentan una tasa mayor de complicaciones postoperatorias, que pudiera reducirse al aportar soporte nutricional preoperatorio.

Agradecimientos: Drs. Hernán de la Fuente, Enrique Waugh, Nicolás Kerrigan, personal paramédico y administrativo que ayudaron a la incorporación y seguimiento de pacientes.

\section{Referencias}

1. Stratton RJ, Green CJ, Elia M. Disease-related malnutrition: an evidence-based approach to treatment. Wallingford: CABI; 2003.

2. Fearon K, Strasser F, Anker SD, Bosaeus I, Bruera E, Fainsinger RL, et al. Definition and classification of cancer cachexia: an international consensus. Lancet Oncol 2011; 12: 489-95.

3. Planas VM and Camarero GE, Importancia de la nutrición en el paciente oncológico. Barcelona: Novartis Consumer Health S.A. 2003.

4. Cid Conde L, Fernández López T, Neira Blanco P, Arias Delgado J, Varela Correa JJ, Gómez Lorenzo FF. Prevalencia de desnutrición en pacientes con neoplasia digestiva previa cirugía. Nutr Hosp 2008; 23 (1): 46-53.

5. Senesse P, Assenat E, Schneider S, Chargari C, Magné $\mathrm{N}$, Azria D, et al. Nutritional support during oncologic treatment of patients with gastrointestinal cancer: Who could benefit? Cancer Treat Rev 2008; 34 (6): 568-75. 34: p. 568-75. 
6. McMillan DC. Systemic inflammation, nutritional status and survival in patients with cancer. Curr Opin Clin Nutr Metab Care 2009; 12 (3): 223-6.

7. Fearon KC. Cancer cachexia: developing multimodal therapy for a multidimensional problem. Eur J Cancer. 2008; 44 (8): 1124-32.

8. Rizk NP, Bach PB, Schrag D, Bains MS, Turnbull AD, Karpeh M, et al. The impact of complications on outcomes after resection for esophageal and gastroesophageal junction carcinoma. J Am Coll Surg 2004; 198 (1): 42-50.

9. McArdle CS, McMillan DC, Hole DJ. Impact of anastomotic leakage on long-term survival of patients undergoing curative resection for colorectal cancer. Br J Surg 2005; 92 (9): 1150-4.

10. Law WL, Choi HK, Lee YM, Ho JW. The impact of postoperative complications on long-term outcomes following curative resection for colorectal cancer. Ann Surg Oncol. 2007; 14 (9): 2559-66.

11. Klidjian AM, Foster KJ, Kammerling RM, Cooper A, Karran SJ. Relation of anthropometric and dynamometric variables to serious postoperative complications. $\mathrm{Br}$ Med J 1980; 281 (6245): 899-901.

12. Putwatana $\mathrm{P}$, Reodecha $\mathrm{P}$, Sirapo-ngam $\mathrm{Y}$, Lertsithichai P, Sumboonnanonda K. Nutrition screening tools and the prediction of postoperative infectious and wound complications: comparison of methods in presence of risk adjustment. Nutrition 2005; 21 (6): 691-7.

13. Sungurtekin H, Sungurtekin U, Balci C, Zencir M, Erdem E. The influence of nutritional status on complications after major intraabdominal surgery. J Am Coll Nutr 2004; 23 (3): 227-32.

14. Gibbs J, Cull W, Henderson W, Daley J, Hur K, Khuri SF. Preoperative serum albumin level as a predictor of operative mortality and morbidity: results from the National VA Surgical Risk Study. Arch Surg 1999; 134 (1): 36-42.

15. Bozzetti F, Gianotti L, Braga M, Di Carlo V, Mariani L. Postoperative complications in gastrointestinal cancer patients: The joint role of the nutritional status and the nutritional support. Clin Nutr 2007; 26 (6): 698-709.

16. Starker PM, Lasala PA, Askanazi J, Gump FE, Forse RA, Kinney JM. The response to TPN. A form of nutritional assessment. Ann Surg 1983; 198 (6): 720-4.

17. Beck AM, Balknäs UN, Camilo ME, Fürst $P$, Gentile MG, Hasunen K, et al. Practices in relation to nutritional care and support report from the Council of Europe. Clin Nutr 2002; 21 (4): 351-4.

18. Correia MI, Waitzberg DL. The impact of malnutrition on morbidity, mortality, length of hospital stay and costs evaluated through a multivariate model analysis. Clin Nutr 2003; 22 (3): 235-9.
19. Askanazi J, Hensle TW, Starker PM, Lockhart SH, LaSala PA, Olsson C, et al. Effect of immediate postoperative nutritional support on length of hospitalization. Ann Surg 1986; 203 (3): 236-9.

20. Bozzetti F, Braga M, Gianotti L, Gavazzi C, Mariani L. Postoperative enteral versus parenteral nutrition in malnourished patients with gastrointestinal cancer: a randomised multicentre trial. Lancet 2001; 3: 358 (9292): 1487-92.

21. Thoresen L, Frykholm G, Lydersen S, Ulveland H, Baracos V, Prado CM, et al. Nutritional status, cachexia and survival in patients with advanced colorectal carcinoma. Different assessment criteria for nutritional status provide unequal results. Clin Nutr 2013; 32 (1): 65-72.

22. Ottery FD. Definition of standardized nutritional assessment and interventional pathways in oncology. Nutrition 1996; 12: 15-9.

23. Murry DJ, Riva L, Poplack DG. Impact of nutrition on pharmacokinetics of antineoplastic agents. Int J Cancer Suppl 1998; 11: 48-51. Review.

24. Thoresen L, Fjeldstad I, Krogstad K, Kaasa S, Falkmer UG. Nutritional status of patients with advanced cancer: the value of using the subjective global assessment of nutritional status as a screening tool. Palliat Med. 2002; 16 (1):33-42.

25. Gupta D, Lis CG, Granick J, Grutsch JF, Vashi PG, Lammersfeld CA. Malnutrition was associated with poor quality of life in colorectal cancer: a retrospective analysis. J Clin Epidemiol 2006; 59 (7): 704-9.

26. Persson C, Sjödén PO, Glimelius B. The Swedish version of the patient- generated subjective global assessment of nutritional status: gastrointestinal vs urological cancers. Clin Nutr 1999; 18 (2): 71-7.

27. Slaviero KA, Read JA, Clarke SJ, Rivory LP. Baseline nutritional assessment in advanced cancer patients receiving palliative chemotherapy.Nutr Cancer. 2003; 46 (2): 148-57.

28. Laky B, Janda M, Cleghorn G, Obermair A. Comparison of different nutritional assessments and body-composition measurements in detecting malnutrition among gynecologic cancer patients. Am J Clin Nutr 2008; 87 (6): 1678-85.

29. Leuenberger M, Kurmann S, Stanga Z. Nutritional screening tools in daily clinical practice: the focus on cancer. Support Care Cancer 2010; 18 Suppl 2: S17-27.

30. Gospodarowicz MK, Miller D, Groome PA, Greene FL, Logan PA, Sobin LH, The Process for Continuous Improvement of the TNM Classification. Cancer 2004; 100 (1): 1-5. Review.

31. Frisancho AR. New norms of upper limb fat and muscle areas for assessment of nutritional status Am J Clin Nutr 
1981; 34 (11): 2540-5.

32. Fearon KC, Voss AC, Hustead DS; Cancer Cachexia Study Group. Definition of cancer cachexia: effect of weight loss, reduced food intake, and systemic inflammation on functional status and prognosis. Am J Clin Nutr 2006; 83 (6): 1345-50.

33. Tan BH, Birdsell LA, Martin L, Baracos VE, Fearon KC. Sarcopenia in an over- weight or obese patient is an adverse prognostic factor in pancreatic cancer. Clin Cancer
Res. 2009; 15 (22): 6973-9.

34. Prado CM, Lieffers JR, McCargar LJ, Reiman T, Sawyer MB, Martin L, et al. Prevalence and clinical implications of sarcopenic obesity in patients with solid tumours of the respiratory and gastrointestinal tracts: a population-based study. Lancet Oncol 2008; 9 (7): 629-35.

35. Arends J, Bodoky G, Bozzetti F, Fearon K, Muscaritoli M, Selga G, et al. ESPEN Guidelines on Enteral Nutrition: Non-surgical oncology. Clin Nutr 2006; 25 (2): 245-59. 